\title{
Constraining (shifting) types at the interface ${ }^{1}$
}

Ethan POOLE - University of California, Los Angeles

\begin{abstract}
This paper argues that traces only range over individual semantic types and cannot be type shifted into higher types to circumvent this restriction. The evidence comes from movement targeting positions where DPs must denote properties and the behavior of definite descriptions in these positions. These constraints on possible traces demonstrate that syntactic operations impose active restrictions on permissible semantic types in natural language.
\end{abstract}

Keywords: semantic types, traces, movement, reconstruction, type shifting, properties.

\section{Introduction}

A longstanding problem in semantics is that natural language only makes use of a small subset of the possible semantic types that are generated by the standard recursive definition in (1).
(1) a. $e$ and $t$ are types;
b. If $\sigma$ and $\tau$ are types, then $\langle\sigma, \tau\rangle$ is a type;
c. Nothing else is a type.

This paper argues for the hypothesis that, while types are in principle unconstrained in the semantics, syntactic operations only make use of a small set of those possible types and thus impose an active constraint on permissible semantic types. I present a case study on movement, in particular on what constitutes a possible trace, i.e. the $\lambda$-bound variables that movement dependencies can map onto. The novel evidence for this study comes from the domain of property-denoting DPs. The argumentation breaks down into two claims. The first claim is that traces only range over individual semantic types, such as entities $(e)$ and degrees $(d)$. Even though natural language has expressions over higher types, like properties $(\langle e, t\rangle)$ and generalized quantifiers $(\langle e t, t\rangle)$, these expressions cannot be represented as traces. I call this the Trace Interpretation Constraint, given in (2) (see also Chierchia, 1984; Landman, 2006).

\section{(2) Trace Interpretation Constraint}

$*\left[\mathrm{DP}_{1} \lambda f_{\sigma} \ldots\left[\ldots\left[f_{\sigma}\right]_{1} \ldots\right]\right]$, where $\sigma$ is not an individual type

The second claim is that the Trace Interpretation Constraint cannot be circumvented by type shifting an individual-type trace into a higher type. I call this the Trace Rigidity Principle, given in (3) (see also Landman, 2004).

\section{(3) Trace Rigidity Principle}

Traces cannot be type shifted.

\footnotetext{
${ }^{1}$ This paper stems from my dissertation work in Poole (2017a). For helpful discussion on the specific issues in this paper, many thanks to Rajesh Bhatt, Kyle Johnson, Barbara Partee, and Ellen Woolford, in addition to Daniel Altshuler, Dylan Bumford, Danny Fox, Stefan Keine, Angelika Kratzer, Andrew McKenzie, and audiences at GLOW 40, Sinn und Bedeutung 22, UMass, and UCLA. This work was supported by the National Science Foundation Graduate Research Fellowship under NSF DGE-1451512.
} 
These constraints together conspire to force movement either to map onto a trace ranging over an individual type (4) or to reconstruct by putting the moved expression back in its launching site at LF (5). All other representations are ill-formed. Thus, the interpretation of movement is tightly restricted, which in turn constrains the actively used semantic types.
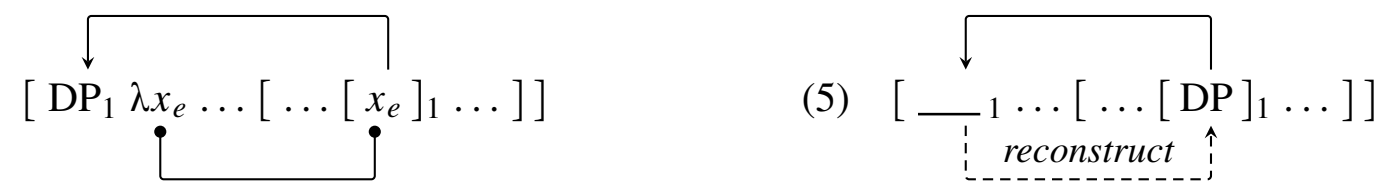

Addressing constraints on permissible semantic types might appear to (and arguably, traditionally does) belong to the domain of lexical items, namely what the semantic types of possible lexical items are. A premise tacitly defended in this paper is that a theory of permissible semantic types must be based on the semantic types of syntactic constituents, which include both lexical items and complex constituents. In simple cases, the possible types of these two coincide; for example, a verb phrase has the same type as an intransitive verb, $\langle e, t\rangle$, irrespective of its internal structure. However, movement would prima facie have the ability to create constituents whose semantic type would not correspond to any known lexical items. Thus, a theory of possible lexical items is not in and of itself a sufficient theory of permissible semantic types. Rather, it must be coupled with a theory of possible traces, i.e. which of the logically possible movement structures are allowed by the grammar, which is what this paper provides.

\section{Trace Interpretation Constraint}

DPs come in three semantic guises (Partee, 1986): entities (type $e$ ), properties (type $\langle e, t\rangle$ ), and generalized quantifiers (type $\langle e t, t\rangle) .{ }^{2}$ There is abundant evidence that entity traces exist, as these are the canonical traces left by movement types like QR. With respect to generalized-quantifier traces, Romero (1998) and Fox (1999) have shown that such traces are unavailable (contra Rullmann, 1995; Cresti, 1995), based on evidence from the correlation between Condition C connectivity and scope reconstruction. Additional arguments against generalized-quantifier traces based on ACD, extraposition, and parasitic gaps can be found in Poole (2017a: 122-126).

(6)

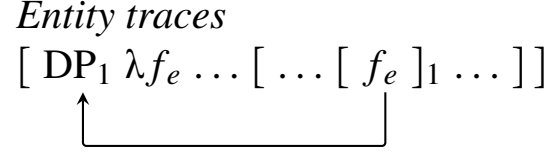

(7) Generalized-quantifier traces

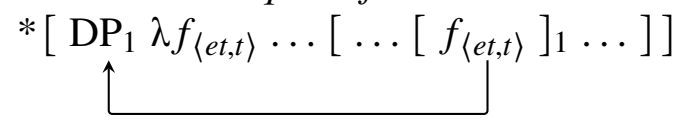

It has not yet been addressed whether property traces exist. ${ }^{3}$ Thus, a central contribution of this project is an empirically motivated argument against property traces (see also Poole, 2017a, b).

(8) Property traces

$*\left[\mathrm{DP}_{1} \lambda f_{\langle e, t\rangle} \ldots\left[\ldots\left[f_{\langle e, t\rangle}\right]_{1} \ldots\right]\right]$

\footnotetext{
${ }^{2}$ Properties are intensional, i.e. $\langle s,\langle e, t\rangle\rangle$, but throughout this paper, I will treat them in purely extensional terms for the sake of simplicity. This reduces them to sets of entities.

${ }^{3}$ Chierchia (1984) argues that property variables exist based on anaphora like such and do so. However, Landman (2006) shows that these cases should be reanalyzed as referring to kinds and do not involve property variables.
} 
This investigation thus supplies the crucial final piece of the argument that the constraint on possible traces is against any higher-type trace (9). This is an important advance in our understanding of the syntax-semantics interface.

\section{(9) Trace Interpretation Constraint}

* $\left[\mathrm{DP}_{1} \lambda f_{\sigma} \ldots\left[\ldots\left[f_{\sigma}\right]_{1} \ldots\right]\right]$, where $\sigma$ is not an individual type

The crucial motivation for the ban on property traces comes from a series of original observations about what I call П-positions. These are syntactic environments where a DP denotes a property (type $\langle e, t\rangle$ ). The four П-positions that form the investigation's empirical base are the pivot of an existential construction (10a), the color term of a change-of-color verb (10b), the name argument of a naming verb (10c), and predicate nominals (10d). Despite their surface heterogeneity, what these four environments have in common is that they require a property-type DP.

\section{$\Pi$-positions}

a. There is $[\text { a potato }]_{\langle e, t\rangle}$ in the pantry.

Existential constructions

b. Megan painted the house [ magenta $]_{\langle e, t\rangle}$.

Change-of-color verbs

c. Irene called the cat $[\text { Snowflake }]_{\langle e, t\rangle}$.

Naming verbs

d. Erika became $[\text { a teacher }]_{\langle e, t\rangle}$.

Predicate nominals

For reasons of space, I do not review the arguments that DPs in these positions denote properties. The arguments, however, come from the respective literatures on each of the $\Pi$-positions and are thus independent from the arguments made here. For change-of-color verbs, the color term denotes a property because these verbs are textbook examples of resultatives (e.g. Kratzer, 2005). For predicates nominals, them being properties is the standard analysis (e.g. Williams, 1983; Partee, 1986). For existential constructions and naming verbs, the arguments are more involved and come from McNally $(1992,1997)$ and Matushansky $(2008)$ respectively. As a bibliographic note, I use "П-positions" as a theory-neutral term because these positions belong to a larger syntactic puzzle observed by Postal (1994), which involves more environments and more movement types than are discussed here. For more about $\Pi$-positions in the context of Postal's puzzle, see Poole (2017a).

The argumentation in this section proceeds as follows: First, I set the stage by showing that movement types in English differ with respect to whether they shift scope, i.e. whether they can reconstruct. Second, I apply these movement types to П-positions, showing that only movement that reconstructs can target them, which categorically precludes some movement types. Third, I argue that the Trace Interpretation Constraint derives this pattern, from which I conclude that property traces do not exist.

\subsection{Movement and scope shifting}

For movement to shift scope means that at LF, the moved DP takes scope in the position achieved by movement. For all overt forms of movement, this corresponds to the DP's surface syntactic 
position. If movement does not shift scope, the scope of the moved DP at LF mismatches its surface position in that it takes scope in its position prior to movement, viz. its base-generated position. This dichotomy is schematized in (11) and (12) where the check mark represents the moved DP's position at LF.

(11)

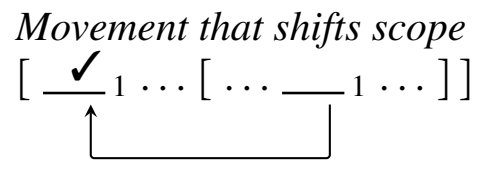

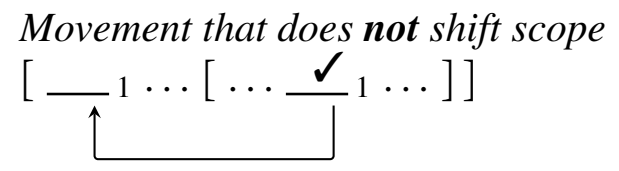

Against this backdrop, let us consider topicalization, wh-movement, and QR.

Topicalization: Topicalization in English obligatorily shifts the scope of the moved DP. ${ }^{4}$ To illustrate, first consider the possible interpretations of the baseline sentence in (13), which has narrow-scope and wide-scope readings of some student with respect to every teacher.

(13) Every teacher likes some student in the first week.

a. Narrow-scope reading

For every teacher $x$, there is some student $y$ such that $x$ likes $y$.

b. Wide-scope reading

There is some student $y$ such that for every teacher $x, x$ likes $y$.

Crucially, in a scenario where the student is a different student for each teacher, only the narrowscope reading in (13a) is true. Topicalizing some student, as in (14), bleeds the narrow-scope reading in (13a).

[Some student $]_{1}$, every teacher likes 1 in the first week.

$$
* \forall \gg \exists ;{ }^{\vee} \exists \gg \forall
$$

The only possible interpretation of (14) is the wide-scope reading, where some student takes scope in the landing site of topicalization, above every teacher. Consequently, (14) is true iff there is a single student that every teacher likes. In sum, topicalization obligatorily shifts scope and does not allow reconstruction.

Wh-movement: Wh-movement optionally shifts the scope of the moved DP. In order to probe scope in constituent questions, we will use how many-questions because, in addition to the wh-meaning component, how many independently carries its own existential quantification that can vary in scope (Kroch, 1989; Cresti, 1995; Rullmann, 1995). Consider the how many-question in (15). Under the wide-scope, de re reading (15a), it is assumed that there is a certain set of books that Nina should read; the speaker is asking how many such books there are. A possible answer to the wide-scope reading is: 'Three books, namely Aspects, Lectures on Government

\footnotetext{
${ }^{4} \mathrm{~A}$ few disclaimers are in order: First, this behavior is notably distinct from other movement types called "topicalization" in other languages, e.g. German V2-fronting, which are indeed able to reconstruct. Second, topicalization is the name of a movement type and should not be conflated with topichood. Third, when investigating English topicalization, there are a number of factors that must be controlled for, which I gloss over here in the interest of space. See Poole (2017a: 48-51) for a more in-depth discussion which shows that the relevant facts hold even when the necessary controls are in place.
} 
and Binding, and The Minimalist Program'. Under the narrow-scope, de dicto reading (15b), there is no assumption that there are any specific books that Nina should read. Rather, it is assumed that she should read a certain number of books, without having any particular books in mind. A possible answer to the narrow-scope reading is: 'Three books, any three'.

(15) [ How many books $]_{1}$ should Nina read 1 this summer?

a. Wide-scope reading how many $\gg$ should For what number $n$ : There are $n$-many particular books $x$ such that Nina should read $x$ this summer.

b. Narrow-scope reading should $\gg$ how many For what number $n$ : It is necessary for there to be $n$-many books $x$ such that Nina reads $x$ this summer.

The wide-scope and narrow-scope readings of (15) can be paraphrased as the questions in (16a) and (16b) respectively.

(16) a. Wide-scope paraphrase of (15)

How many books are there that Nina should read this summer?

b. Narrow-scope paraphrase of (15)

What is the number such that Nina should read that many books this summer?

The scope ambiguity in (15) is the result of the fact that wh-movement only optionally shifts scope and thus allows a reconstructed derivation.

Quantifier Raising: By definition, QR cannot reconstruct. However, as a disclaimer, we must distinguish $\mathrm{QR}$ for scope shifting and $\mathrm{QR}$ for interpreting quantifiers. Although these two functions of QR ordinarily coalesce (at least in English), we will see that this does not hold for $\Pi$-positions: quantificational DPs can occur in П-positions, but they do not enjoy the scopal mobility that QR would afford. For reasons of space, I do not discuss the issue of how to interpret quantificational DPs in П-positions if not by QR. It is essentially an open question, though see Poole (2017a: 83-87) for discussion and some possible solutions.

\section{2. П-positions}

The Trace Interpretation Constraint makes the two predictions about П-positions in (17). This section shows that both of these predictions bear out for the four П-positions.

(17) a. Scope prediction

If movement targets a П-position, it must reconstruct, because an entity trace is type-incompatible with a property-denoting DP.

b. Movement-type-prediction

If a movement type cannot reconstruct, it can never target $\Pi$-positions. 
Existential constructions: Wh-movement can target the pivot of an existential construction (18b), but topicalization (18c) and QR cannot (18d). ${ }^{5}$ This confirms the movement-type prediction for existential constructions, because the two movement types that cannot shift scope, topicalization and QR, cannot target the П-position.

(18) a. There is a potato in the pantry.

Baseline

b. ${ }^{\checkmark}$ What $_{1}$ is there 1 in the pantry?

Wh-movement

c. $*[\text { A potato }]_{1}$, there is 1 in the pantry.

Topicalization

d. There must be someone in his house.

$Q R:{ }^{`}$ must $\gg \exists ; * \exists \gg$ must

Even though $w h$-movement can ordinarily shift scope, when it targets the pivot of an existential construction, scope shifting is rendered impossible, and the movement must reconstruct (19).

[How many questions $]_{1}$ should there be 1 on the exam?

*how many $\gg$ should; ${ }^{\prime}$ should $\gg$ how many

To appreciate this fact, let us compare the existential construction in (19) with its corresponding copula construction in (20), where how many is able to scope above or below should. Paraphrases of the (hypothetical) wide-scope and narrow-scope readings of (19) and (20) are given in (21).

Copula equivalent of (19)

$\checkmark$ how many $\gg$ should; ${ }^{`}$ should $\gg$ how many [ How many questions $]_{1}$ should 1 be on the exam?
a. Narrow-scope paraphrase
$\checkmark$ existential (19); ${ }^{`}$ copula (20)
What is the number such that it is necessary that that many questions be on the exam?
b. Wide-scope paraphrase
*existential (19); ${ }^{`}$ copula (20)
How many questions are there such that it is necessary that they be on the exam?

Consider the appropriateness of (19) and (20) in two different scenarios where I am a TA and the professor is preparing the final exam. In the first scenario, she wants to know the number of questions that I think the exam should have so that the grading is manageable; the identity of the questions does not matter at this point. Both (19) and (20) are appropriate in this context because they both have a narrow-scope reading, as paraphrased in (21a). In the second scenario, the professor has asked me to pick out from a workbook the questions that I think should be on the exam. She wants to know the number of questions that I have selected so that she can gauge the amount of time that the exam room should be reserved for. Thus, she is asking about the cardinality of a set that exists in the actual world, the set of questions that I have picked. While the copula construction in (20) is appropriate in this context, the existential construction in (19) is not. This contrast reflects that (20) but not (19) has a wide-scope reading where how many scopes above should, as paraphrased in (21b). This difference follows from the fact that $w h$-movement cannot shift scope when it targets a $\Pi$-position, thereby forcing a narrow-scope, reconstructed reading of how many. This confirms the scope prediction for existential constructions. Further confirmation of the scope prediction comes from negative

\footnotetext{
${ }^{5}$ The observation that QR cannot target the pivot of an existential construction comes from Williams (1984).
} 
islands, which independently block reconstruction (e.g. Rullmann, 1995). Since negative islands force how many to take wide scope and П-positions force how many to take narrow scope, the two should be mutually exclusive. This prediction is borne out, as shown in (22). ${ }^{6}$
a. * How many books $]_{1}$ aren't there 1 on the table?
b. $\checkmark[\text { How many tables }]_{1}$ aren't there books on 1 ?

Change-of-color verbs: Wh-movement can target the color term of a change-of-color verb (23b), e.g. paint, turn, and dye, but topicalization cannot (23c).

(23) a. Megan painted the house magenta.

Baseline

b. $\checkmark[\text { What color }]_{1}$ did Megan paint the house __ 1 ?

Wh-movement

c. * Magenta 1 , Megan painted the house

Topicalization

There is no general prohibition against topicalizing color terms. A color term can be topicalized if it does not occur with a change-of-color verb (24). The prohibition on topicalization targeting color terms applies exclusively to those color terms that are arguments of change-of-color verbs.

$\{\text { Green / that color }\}_{1}$, he never discussed 1 with me.

[Postal 1994:164]

QR also cannot target the color term of a change-of-color verb (25a), which we can compare with QR targeting the object (25b), which is indeed possible. ${ }^{7}$
a. A (\#different) contractor painted the house every color.
$\checkmark \exists \gg \forall ; * \forall \gg \exists$
b. A (different) contractor painted every house that ugly green.
$\checkmark \exists \gg \forall ; \vee \forall \gg \exists$

(25a) is true iff there is a single contractor, who incidentally did lots of painting, but not if there is a different contractor for each color. This confirms the movement-type prediction for change-of-color verbs. Turning to the scope prediction, when $w h$-movement targets the color term, it must reconstruct. Thus, (26) only has the narrow-scope reading, as paraphrased in (26a), and extraction from negative islands is outright ungrammatical (27), thereby confirming the scope prediction for change-of-color verbs.

(26) [ How many colors $]_{1}$ should Nina paint the house 1 ?

a. ${ }^{\checkmark}$ Narrow-scope paraphrase: What is the number such that it is necessary that Nina paint the house that many colors?

$\checkmark$ should $\gg$ how many

b. *Wide-scope paraphrase: How many colors are there such that it is necessary that Nina paint the house those colors?

*how many $\gg$ should

\footnotetext{
${ }^{6}$ The same fact can be shown with $w h$-islands; see Poole (2017a: 56-59).

${ }^{7}$ I include different to bias towards the inverse-scope reading. The \#-mark indicates that different is infelicitous if the sentence were uttered out-of-the-blue, because it lacks the inverse-scope reading that would require QR. There is a felicitous reading of (25a) in which different is interpreted as different with respect to something previously mentioned in the discourse, e.g. another contractor, but this reading does not involve inverse scope.
} 
(27) a. * [ How many colors $]_{1}$ did no one paint their house __ 1 ?

b. $\checkmark$ How many houses $]_{1}$ did no one paint __ 1 lime green?

Naming verbs: The same pattern is observed for naming verbs and predicate nominals, so here the discussion will be more compact. Wh-movement can target the name argument of a naming verb (28b), e.g. name, call, and baptize, but topicalization (28c) and QR cannot (28d). As with color terms, there is no general prohibition against topicalizing names (29). Finally, when wh-movement targets the name argument, it must reconstruct (30). This confirms the movement-type and scope predictions for naming verbs.

(28) a. Irene called the cat Snowflake.

Baseline

b. $\checkmark[\text { What name }]_{1}$ did Irene call the cat $\_1$ ?

Wh-movement

c. ${ }^{*}$ Snowflake $e_{1}$, Irene called the cat

Topicalization

d. A (\#different) child called the cat every nickname.

$Q R:^{\vee} \exists \gg \forall ; * \forall \gg \exists$

(29) Raphael $_{1}$, we never discussed 1 as a possible name for him.

[Postal 1994:164]

(30) [ How many nicknames $]_{1}$ should Nina call the cat ___ 1 ?

*how many $\gg$ should; ${ }^{\top}$ should $\gg$ how many

Predicate nominals: Wh-movement can target predicate nominals (31b), but topicalization (31c) and QR cannot (31d). Furthermore, when wh-movement targets a predicate nominal, it must reconstruct (32). This confirms the movement-type and scope predictions for predicate nominals.

(31) a. Erika became a teacher.

Baseline

b. $\checkmark[\text { What (kind of teacher) }]_{1}$ did Erika become

Wh-movement

c. * A math teacher $]_{1}$, Erika became ${ }_{1}$.

Topicalization

d. A (\#different) student became every kind of teacher.

$Q R: \sqrt{ } \exists \gg \forall ; * \forall \gg \exists$

\footnotetext{
[ How many kinds of teacher $]_{1}$ should Nina become ___ 1 ?

*how many $\gg$ should; $`$ should $\gg$ how many
}

\subsection{Putting together the pieces}

The previous section confirmed the two predictions of the Trace Interpretation Constraint: (i) if movement targets a П-position, it must reconstruct, because an entity trace is type-incompatible with a property-denoting DP, and (ii) if a movement type cannot reconstruct, it can never target $\Pi$-positions. Descriptively, then, what П-positions reveal is that the semantic representation of scope-shifting movement is incompatible with positions where DPs must denote properties. According to the standard mechanism of interpreting movement (e.g. Heim and Kratzer, 1998), and also the Trace Interpretation Constraint, this follows straightforwardly: the representation of scope-shifting movement involves movement leaving an entity (type $e$ ) trace. Leaving a type- $e$ 
trace would shift scope, but such a trace does not furnish the property meaning required by $\Pi$-positions, yielding ungrammaticality (33). Reconstruction obviates this problem by placing the moved expression back in the launching site of movement at LF. Thus, if a DP would not ordinarily violate the property requirement of П-positions, then it will not do so under reconstruction either (34).

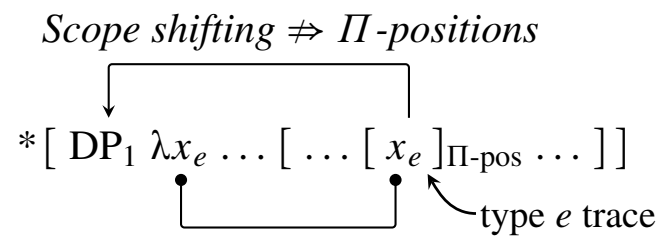

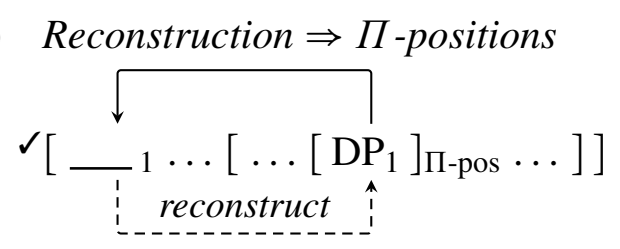

According to this analysis, П-positions are an instance where movement must reconstruct in order to avoid the semantic-type mismatch that would occur if the moved DP were not interpreted in its base-generated position.

Crucially, the ungrammaticality of scope-shifting movement targeting П-positions indicates that movement cannot map onto a trace ranging over properties, where the moved DP denotes either a property or a generalized quantifier over properties, as schematized in (35).

Property traces are ungrammatical

$$
\begin{aligned}
& \text { a. } *\left[\begin{array}{ll}
\mathrm{DP}_{\langle e, t\rangle} & \lambda f_{\langle e, t\rangle}[\ldots f \ldots]
\end{array}\right] \\
& \text { b. } *\left[\mathrm{DP}_{\langle\langle e t, t\rangle, t\rangle} \lambda f_{\langle e, t\rangle}[\ldots f \ldots]\right.
\end{aligned}
$$

Empirically, if (35a) and (35b) were not ungrammatical, they would derive the wrong scope facts (see above). Moreover, even in instances that involve apparent quantification over properties, these quantifiers over properties cannot take scope over other scope-bearing elements in the sentence, as shown in (36) for existential constructions.

(36) a. There wasn't every kind of doctor at the convention. ' not $\gg$ every; *every $\gg$ not

b. There wasn't only one kind of doctor at the convention.

$\checkmark$ not $\gg$ only one; *only one $\gg$ not

This unavailability of wide-scope is expected if (35b), where a generalized quantifier over properties has undergone $\mathrm{QR}$, is an unavailable representation. Moreover, if a trace ranging over properties is unavailable in (35b), then we can generalize that it is also unavailable in (35a), which completely rules out property traces. ${ }^{8}$ Thus, what the ungrammaticality of scope-shifting movement targeting П-positions ultimately reveals is that the syntax-semantics mapping does not permit movement to map onto traces ranging over properties, in accordance with the Trace Interpretation Constraint.

\footnotetext{
${ }^{8}$ Strictly speaking, (35a) would not affect quantificational scope and therefore can only be ruled out by deduction. However, the alternative analysis, where (35a) is possible but (35b) is not, would require that movement have some way of knowing the semantic type of the moving expression. I do not see how this would be possible under standard conceptions of syntax and its interface with semantics. According to the analysis here, the only trace that movement can leave is an entity trace, and thus all the configurations in (35) are blocked without having to examine the elements involved in the movement chain.
} 


\section{Trace Rigidity Principle}

The Trace Interpretation Constraint raises the possibility that the grammar could use an entity trace, but type shift that trace into a higher type, e.g. rendering it compatible with П-positions. This section argues that such a rescue procedure does not happen and that traces cannot be type shifted, a principle which I call the Trace Rigidity Principle in (37) (or 'trace rigidity' for short).

\section{(37) Trace Rigidity Principle}

Traces cannot be type shifted.

Without trace rigidity, the Trace Interpretation Constraint would effectively be vacuous and unobservable because it could always be circumvented under the surface. Because the Trace Interpretation Constraint can in fact be observed, there is already reason to believe that trace rigidity holds. However, what this section argues is that there is independent evidence for trace rigidity. I show that anaphoric definite descriptions, a superset of traces under Trace Conversion, cannot occur in $\Pi$-positions, but their nonanaphoric counterparts can. Thus, we are able to view the effects of (37) outside the context of movement. I then argue that it is anaphoric definites that cannot be type shifted and develop a syntactic analysis of this incompatibility in terms of the weak-strong definite distinction (in the sense of Schwarz, 2009). The upshot of this proposal is that trace rigidity follows from how DPs are constructed in the syntax.

The point of departure is the observation that at first glance, seemingly type-e elements appear to be able to occur in $\Pi$-positions (38). Given the fact that $\Pi$-positions require property-denoting expressions, why are the examples in (38) grammatical?

(38) a. Megan painted the house that hideous shade of purple.

Change-of-color verbs

b. Irene called the cat that dumb nickname.

Naming verbs

c. Erika became that kind of teacher.

Predicate nominals

\subsection{Type shifting to property}

Partee (1986) proposes a set of semantic type shifters that allow DPs to flexibly shift from one of the three possible types to another. The type shifters that are important for our purposes, because they allow shifting into the property domain, are IDENT, PRED, and $\mathcal{B E}$ (39).

$$
\begin{aligned}
\text { a. } & \text { IDENT: } j \rightarrow \lambda x \cdot x=j \\
\text { b. } & \text { PRED: } x \rightarrow{ }^{\cup} x \\
\text { c. } & \mathcal{B E}: \mathcal{P} \rightarrow \lambda x . \mathcal{P}([\lambda y \cdot y=x]) \\
& \mathcal{P} \rightarrow \lambda x .\{x\} \in \mathcal{P}
\end{aligned}
$$

The functor IDENT is a total function that maps any element onto its singleton set. The functor PRED maps the entity-correlate of a property onto the corresponding property (Chierchia, 1984). For example, PRED maps $\llbracket$ goodness $\rrbracket$ to $\llbracket \operatorname{good} \rrbracket$ and $\llbracket$ green $\rrbracket$ the noun to $\llbracket$ green $\rrbracket$ the adjective. 
$\mathcal{B E}$ is a homomorphism between $\langle e t, t\rangle$ and $\langle e, t\rangle$. It applies to a generalized quantifier, finds all of the singleton sets therein, and collects the elements of these singleton sets into a set. For more discussion of these type shifters in the context of П-positions, see Poole (2017a: 199-204).

I propose that DPs never start out denoting properties. ${ }^{9}$ A property denotation is always achieved by type shifting from an individual denotation $(e)$ or a generalized-quantifier denotation $(\langle e t, t\rangle)$. Consequently, П-positions require a type shifter for the structure to semantically compose, as schematized in (40), because they require property-type DPs. For the sake of simplicity, I will generally assume that the type shifter used is $\mathcal{B E}$, though nothing critical hinges on this.

(40) a. Existential constructions

There is $[\mathcal{B E}($ a potato $)]$ in the pantry.

$$
\langle e t, t\rangle \rightarrow\langle e, t\rangle
$$

b. Change-of-color verbs

Megan painted the house $[\operatorname{PRED}($ magenta $)]$.

$$
e \rightarrow\langle e, t\rangle
$$

c. Naming verbs

Irene called the cat $[\mathcal{B E}($ Snowflake $)]$.

$\langle e t, t\rangle \rightarrow\langle e, t\rangle$

d. Predicate nominals

Erika became $[\mathcal{B E}($ a teacher $)]$.

$$
\langle e t, t\rangle \rightarrow\langle e, t\rangle
$$

Let us take stock and look ahead. We now have an explanation for why seemingly type- $e$ (and $\langle e t, t\rangle)$ expressions can occur in $\Pi$-positions: they are type shifted into property meanings. However, thus far, nothing prevents these same type shifters from applying to traces, circumventing the Trace Interpretation Constraint. The next subsection introduces another generalization about П-positions: they prohibit anaphoric definite descriptions. I argue that the ban on anaphoric definites and the ban on scope-shifting movement from $\Pi$-positions are one and the same under Trace Conversion, wherein traces are anaphoric definite descriptions. I then propose a syntactic account of the complementarity of type shifting and anaphoric definites.

\section{2. П-positions prohibit anaphoric definites}

While some type- $e$ expressions can occur in П-positions as a result of property denotations being derived via type shifting, it is not the case that П-positions permit all type-e expressions. As such, this means that not all expressions can type shift into property denotations. This section observes that $\Pi$-positions prohibit anaphoric definite descriptions (41). Thus, it must be the case that anaphoric definites cannot be type shifted to type $\langle e, t\rangle$. Following Schwarz's (2009) terminology, I will call anaphoric definite descriptions strong definites and nonanaphoric definite descriptions weak definites.

\section{(41) Definite generalization}

$\Pi$-positions prohibit anaphoric (= strong) definite descriptions.

Testing for the felicity of strong definites in П-positions requires some amount of indirect

\footnotetext{
${ }^{9}$ Poole (2017a: 203-204) suggests that this may explain why property DPs seem to be marked crosslinguistically.
} 
reasoning, which is worth spelling out explicitly. Examples like (38) show that definites are in principle allowed in П-positions, but they do not reveal what kinds of definites. It is possible to create contexts where only a strong definite would be felicitous. There are two properties that distinguish strong definites from weak definites, which can be used to create such contexts: (i) strong definites must have an antecedent and (ii) they do not have to satisfy the standard uniqueness requirement of (weak) definites (Schwarz, 2009). When these two conditions are satisfied and controlled for, definites become unacceptable in П-positions. Because definites can occur in П-positions, but not in these contexts that allow only strong definites, we can reason that it must be the case that the definites in П-positions are necessarily weak definites. With this logic in mind, I show three pieces of evidence below that support the generalization in (41). ${ }^{10}$

First, a strong definite can refer to a previously mentioned indefinite. In (42a), the definite the shade, or even the color, can refer back to the indefinite $a$ shade of red. In this context, there may be multiple shades or colors that Dorothy finds too dark or other colors that Blanche picked out. Thus, it is not the case that the shade and the color are conveying their referent based on uniqueness. As shown in (42b), a definite description in a П-position (here, a change-of-color verb) in the same context is infelicitous. What this infelicity discloses is that the definite in (42b) must be a weak definite and its uniqueness requirement is not being satisfied.

(42) Blanche picked out a shade of red for the living room.

Color verbs
a. ${ }^{\checkmark}$ But Dorothy thought that the shade/color was too dark.
b. \# And Dorothy painted the room [ the shade/color $]_{\Pi-p o s}$.

The second piece of evidence is that a strong definite can covary with an indefinite in a quantificational sentence. For example, in (43a), the color or the shade can covary with a color, even though the situations being quantified over, Irene picking out colors, presumably contain more than one color and thus would not satisfy the uniqueness requirement. This kind of covariance requires an anaphoric relationship with the quantifier, which a weak definite cannot achieve. As shown in (43b), a definite description in a П-position (here, a change-of-color verb) in the same context is infelicitous. As above, this infelicity indicates that the definite in (43b) must be a weak definite and its uniqueness requirement is not being satisfied.

(43) Every time Irene picks out a color for the bathroom, ...

Color verbs
a. ${ }^{`}$ Helen complains that the color/shade is too bright.
b. \#Helen has to paint the room [ the color/shade $]_{\Pi \text {-pos. }}$.

(44)-(46) show that the same contrast holds for the other П-positions as well.

(44) In every hotel room with an ugly lamp, ...

Existential constructions

a. the lamp is on the dresser.

b. \#there is [ the lamp $]_{\Pi-p o s}$ on the dresser.

\footnotetext{
${ }^{10}$ Not all of the data is given here, for reasons of space; see Poole (2017a: 204-212).
} 
(45) Every time that my mom found a new puppy name, ...

Naming verbs

a. ' my dad vetoed the name.

b. \# she nicknamed the family dog $[\text { the name }]_{\Pi-p o s}$.

(46) In every store with a rare type of plant,...

Predicate nominals

a. ' my aunt bought the rare type.

b. \# my aunt bought a plant that was $[\text { the rare type }]_{\Pi-p o s}$.

The third piece of evidence is that while the previous two sets of examples show that strong definites are ungrammatical in П-positions, the inverse can likewise be observed: weak definites are grammatical in П-positions. There are certain contexts that require a weak definite. One such context is bridging contexts where there is a part-whole relation between a definite description and the individuals and events in the preceding discourse, which is sufficient to satisfy the uniqueness requirement of the (weak) definite (Schwarz, 2009). (47) shows that part-whole bridging contexts allow definite descriptions in $\Pi$-positions (here, an existential construction).

(47) A: What did you like about the fridge?

Existential constructions

B: Well, there was $[\text { the spacious vegetable crisper }]_{\Pi-p o s}$.

We now have two generalizations about what is not allowed in $\Pi$-positions: the scope generalization (48a), which reduces to an incompatibility with type- $e$ traces, the only type of trace available according to the Trace Interpretation Constraint, and the definite generalization (48b).

\section{a. Scope generalization}

Movement that shifts scope cannot target П-positions.

\section{b. Definite generalization}

$\Pi$-positions prohibit anaphoric (= strong) definite descriptions.

I propose that these two generalizations are one and the same because "traces" are in fact anaphoric definite descriptions, i.e. strong definites. The idea that traces are related to anaphoric definite descriptions is quite old; see Engdahl's $(1980,1986)$ early work on the semantics of questions. However, the idea is best known now as Trace Conversion, according to which downstairs copies of moved DPs are rendered interpretable at LF by converting them into definite descriptions with a variable (49) (Sauerland, 1998, 2004; Fox, 1999, 2002, 2003).

\footnotetext{
a. Standard traces

$[\text { every cat }]_{1} \lambda x\left[\right.$ a child adopted $\left.\left.\left[t_{x}\right]_{1}\right]\right]$

b. Traces as anaphoric definites

$\left[[\text { every cat }]_{1} \lambda x\left[\right.\right.$ a child adopted $\left.\left.[\text { the cat } x]_{1}\right]\right]$
}

The technical apparatus performing this operation is a special LF rule that comprises two parts: insertion of a variable (50a) and determiner replacement (50b). The inserted variable is bound by the $\lambda$-abstraction introduced below the landing site of movement. 
a. Variable Insertion

(Det) Pred $\rightarrow($ Det) $[[$ Pred $][\lambda y \cdot y=g(n)]] \quad$ (where $g$ is the assignment function)

b. Determiner Replacement

(Det) $[[$ Pred $][\lambda y \cdot y=g(n)]] \rightarrow$ the $[[$ Pred $][\lambda y \cdot y=g(n)]]$

The result of Trace Conversion is that traces are anaphoric definite descriptions, which allows the scope generalization to be subsumed under the definite generalization. Accordingly, the Trace Rigidity Principle can be recast as (51) to encompass this combined generalization.

\section{(51) Trace Rigidity Principle (revised)}

Traces cannot be type shifted.

$\leadsto$ Anaphoric definite descriptions cannot be type shifted.

\subsection{Anaphoric definites and type shifting}

Under the revised version of the Trace Rigidity Principle in (51), the question of why strong definites (including traces) are disallowed in П-positions translates into the question of why strong definites cannot be type shifted into property denotations. One possibility that can be immediately set aside is linking the incompatibility directly to anaphoricity. Many of the infelicitous examples of definite descriptions in П-positions are improved when the is replaced with that, as illustrated in (52) with a change-of-color verb.

(52) Blanche picked out a shade of red for the living room.

a. \# And Dorothy painted the room [ the shade/color $]_{\Pi \text {-роs. }}$.

b. $\checkmark$ And Dorothy painted the room [ that shade/color $]_{\Pi \text {-pos }}$.

While a definite description the NP cannot establish an anaphoric relation in a П-position (52a), that $N P$ can do so (52b). It is not entirely clear where that $N P$ fits within the strong/weak definite distinction, but (52) nevertheless shows that anaphoricity alone cannot be responsible for trace rigidity. Rather, it must be something specific about definite descriptions with the determiner the. In this section, I develop a syntactic analysis of the Trace Rigidity Principle, capitalizing on one aspect that has been argued to differ between strong and weak definites: their determiners.

Schwarz (2009) proposes that the strong/weak definite distinction results from having two separate definite determiners (53). In (53), I provide Schwarz's more standard denotations that return an expression of type- $e$ and also denotations that return a generalized quantifier. ${ }^{11}$ Both determiners are associated with uniqueness, represented by the l-operator. However, the strongdefinite determiner also has an index (53). The anaphoricity of the strong-definite determiner derives from the index, which can be bound or valued contextually in the same manner as a pronoun, thereby picking out a particular referent rather than relying on uniqueness alone.

\footnotetext{
${ }^{11}$ Schwarz's (2009) denotations are intensional and include a situation variable. As I have been assuming an extensional system, I have simplified the denotations.
} 
(53)

Schwarz's (2009) weak and strong definite determiners

a. $\quad\left[\right.$ the $_{\mathrm{WEAK}} \rrbracket=\lambda \mathrm{P}_{\langle e, t\rangle} \cdot \iota x[\mathrm{P}(x)]$

$$
=\lambda \mathrm{P}_{\langle e, t\rangle} \lambda \mathrm{Q}_{\langle e, t\rangle} \cdot \mathrm{Q}(\iota x[\mathrm{P}(x)])
$$

b. $\quad\left[\right.$ the $_{\text {STRONG }} \rrbracket=\lambda y_{e} \lambda \mathrm{P}_{\langle e, t\rangle} \cdot \mathrm{\iota} x[\mathrm{P}(x) \wedge x=y]$

$$
=\underbrace{\lambda y_{e}}_{\text {index }} \lambda \mathrm{P}_{\langle e, t\rangle} \lambda \mathrm{Q}_{\langle e, t\rangle} \cdot \mathrm{Q}(\iota x[\mathrm{P}(x) \wedge \underbrace{x=y}_{\text {index }}])
$$

In some languages, the weak-definite and strong-definite determiners have unique realizations or are individually subject to special morphological operations. For example, in German, the determiner in weak definites contracts with prepositions (subject to gender and case), but not in strong definites (54) (Schwarz, 2009).

\section{German strong/weak definite distinction}

In jeder Bibliothek, die ein Buch ber Topinambur hat, sehe ich in every library that a book about topinambur has look I

\{\#im / ' in dem $\}$ Buch nach, ob man Topinambur grillen kann. in.the $_{\text {WEAK }}$ in the STRONG $_{\text {book PRT }}$ whether one topinambur grill can

'In every library that has a book about topinambur, I check in the book whether one can grill topinambur.'

[Schwarz 2009:33]

Crucially, Trace Conversion requires the strong-definite determiner in order to establish a connection between the upstairs moved DP and the downstairs definite description. Within the strong/weak definite distinction, Trace Conversion, however, operates somewhat differently. Rather than having two separate rules, one for inserting a variable and another for replacing the determiner, there is only a single rule that replaces the determiner in the downstairs DP with the strong-definite determiner, as this determiner contains the variable, i.e. the index. The index is what is then bound by the $\lambda$-abstraction created by movement, as schematized in (55).

\section{Trace Conversion with the strong-definite determiner}

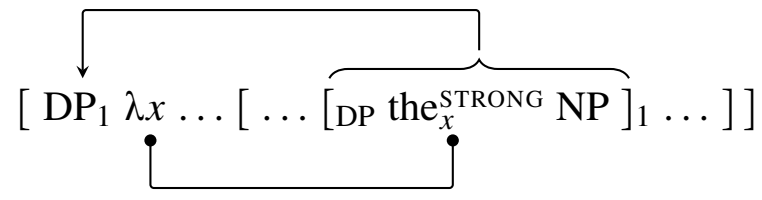

The syntactic analysis of trace rigidity breaks down into two pieces. First, I propose that the weak-definite and strong-definite determiners occupy distinct syntactic positions in the functional structure of a nominal. The strong-definite determiner occupies $\mathrm{D}^{0}(56)$, and the weak-definite determiner occupies some lower functional head, which I label $n^{0}$ for convenience (57). ${ }^{1213}$ In English, $n^{0}$ raises to $\mathrm{D}^{0}$ to form a complex head, which spells out as the regardless of whether $n^{0}$ or $\mathrm{D}^{0}$ is the head that contains the determiner (58).

\footnotetext{
${ }^{12}$ The determiner that might also be in $n^{0}$, explaining why that $N P$ can occur in $\Pi$-positions in anaphoric contexts. ${ }^{13}$ The structures in (56) and (57) might fit into a more articulated nominal structure like that of Zamparelli (2000). For similar proposals that the strong/weak definite distinction is syntactically encoded, see Patel-Grosz and Grosz (2017) and Cheng et al. (2017).
} 
(56)

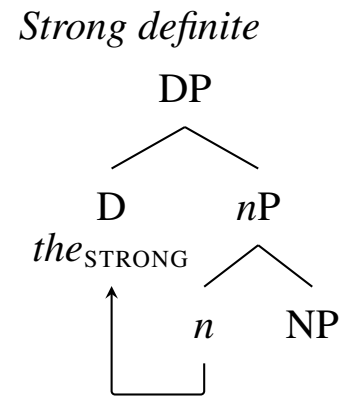

(57)

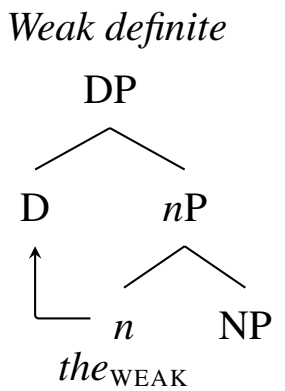

(58)

English Vocabulary Items

a. $\left[\mathrm{D}+\sqrt{\mathrm{THE}}_{\mathrm{WEAK}}\right] \leftrightarrow /$ the $/$

b. $\left[\sqrt{\mathrm{THE}}_{\mathrm{STRONG}}+n\right] \leftrightarrow /$ the $/$

The denotations of the definite determiners in (53) do not permit an $n \mathrm{P}$ headed by the $e_{\mathrm{WEAK}}$ to serve as the semantic argument of the $e_{\text {STRONG }}$. Therefore, a given DP can only contain one of the definite determiners.

The second piece of the proposal is that nominal type shifters also occupy $\mathrm{D}^{0}$, competing with the strong-definite determiner for the same syntactic slot. As such, a DP can either include the strong-definite determiner or a nominal type shifter, but never both. This complementary distribution has two crucial consequences. First, a definite description that has been type shifted is necessarily a weak definite because the only definite determiner that can occur alongside a type shifter is the WEAK $_{\text {(59). }}{ }^{14}$ This accounts for the observation from section 3.2 that definite descriptions in $\Pi$-positions are infelicitous in contexts that only license strong definites and hence are necessarily weak definites.

\section{Type-shifted definites are always weak definites}

a. $\left[\right.$ DP $($ SHIFTER $)\left[n\right.$ P the WEAK $\left._{\text {NP }]]}\right]$ Weak definite; Type shifting possible

b. [DP the STRONG $\left.\left._{n \text { P } n^{0}} \quad \mathrm{NP}\right]\right] \quad \leadsto$ Strong definite; Type shifting impossible

Second, Trace Conversion and type shifting cannot apply to one and the same DP. In a П-position, it is a lose-lose situation. On one hand, if the converted trace contains a type shifter to achieve the required property denotation, the only definite determiner available is the $e_{\text {WEAK }}$, which has no variable for the $\lambda$-abstraction to bind (60). The result is vacuous quantification and thus ungrammaticality. On the other hand, if the converted trace contains the strong-definite determiner, there is a variable for the $\lambda$-abstraction to bind, but the DP does not denote a property and runs afoul of the property requirement of $\Pi$-positions (61). Consequently, because either option results in ungrammaticality, the only option left for movement targeting a $\Pi$-position is to reconstruct.
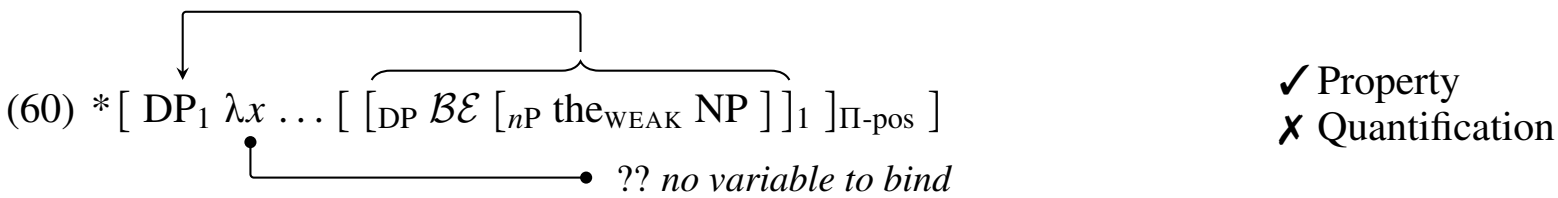

\footnotetext{
${ }^{14}$ To use $\mathcal{B E}$ for weak definites requires that the $e_{\text {WEAK }}$ return a generalized quantifier or that $\mathrm{D}^{0}$ can be $\mathcal{B E} \circ$ LIFT. (53a) provided a denotation for the $e_{\mathrm{WEAK}}$ that returns a generalized quantifier.
} 


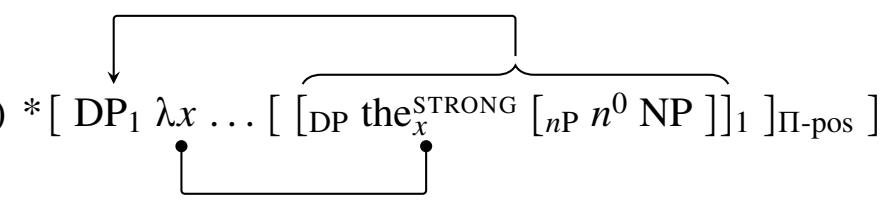

$x$ Property

$\checkmark$ Quantification

This analysis manages to derive both the definite generalization and the scope generalization from one stipulation, namely the complementary distribution of the strong-definite determiner and nominal type shifters. ${ }^{15}$ One might wonder whether there is any independent reason to believe that the strong-definite determiner and nominal type shifters should be in complementary distribution. An idea that floats around in the literature is that English the is an overt type shifter, e.g. a overt l-operator or an overt encoding of the "natural" type shifter $\mathcal{T H \mathcal { E }}$ (e.g. Partee, 1986; Chierchia, 1998). If this were to hold of the strong-definite determiner, then it would compete with the property-yielding type shifters for the $\mathrm{D}^{0}$ slot because it is itself a type shifter.

\section{Conclusion}

In this paper, I have presented a case study on what constitutes a possible trace, arguing for the two following constraints on interpreting movement:

(62) Trace Interpretation Constraint

$*\left[\mathrm{DP}_{1} \lambda f_{\sigma} \ldots\left[\ldots\left[f_{\sigma}\right]_{1} \ldots\right]\right]$, where $\sigma$ is not an individual type

\section{(63) Trace Rigidity Principle}

Traces cannot be type shifted.

The paper began with the problem of the standard recursive definition of semantic types overgenerating. The Trace Interpretation Constraint and the Trace Rigidity Principle demonstrate that movement is one domain in which the grammar only makes use of a small set of the possible types, namely the individual types. I would like to suggest that this is representative of the role that syntactic operations - and perhaps the properties of those operations, like economy-play in restricting the actively used semantic types. That is, while semantic types might be in principle unconstrained in the semantics, they are actively constrained by different modules of grammar.

\section{References}

Cheng, L., C. Heycock, and R. Zamparelli (2017). Two levels for definiteness. Handout from talk presented at GLOW in Asia XI.

Chierchia, G. (1984). Topics in the Syntax and Semantics of Infinitives and Gerunds. Ph. D. thesis, University of Massachusetts, Amherst, MA.

Chierchia, G. (1998). Reference to kinds across language. Natural Language Semantics 6(4), 339-405.

Cresti, D. (1995). Extraction and reconstruction. Natural Language Semantics 3, 79-122.

Engdahl, E. (1980). The Syntax and Semantics of Questions in Swedish. Ph. D. thesis, University of Massachusetts, Amherst, MA.

\footnotetext{
${ }^{15}$ Partee (1986) is agnostic about where type shifters live in the grammar. According to this analysis, at least some type shifters exist in the narrow syntax because they are in complementary distribution with the strong-definite determiner. We might take this as evidence that type shifters exist only in the syntax as determiners and that type shifting is not a last-resort mechanism to repair type mismatches.
} 
Engdahl, E. (1986). Constituent Questions. Dordrecht: D. Reidel Publishing Company.

Fox, D. (1999). Reconstruction, variable binding, and the interpretation of chains. Linguistic Inquiry 30, 157-196.

Fox, D. (2002). Antecedent-contained deletion and the copy theory of movement. Linguistic Inquiry 33(1), 63-96.

Fox, D. (2003). On logical form. In R. Hendrick (Ed.), Minimalist syntax, pp. 82-123. Oxford: Blackwell.

Heim, I. and A. Kratzer (1998). Semantics in Generative Grammar. Oxford: Blackwell.

Kratzer, A. (2005). Building resultatives. In C. Maienborn and A. Wöllstein-Leisten (Eds.), Event Arguments: Foundations and Applications, pp. 177-212. Tübingen: Niemeyer.

Kroch, A. (1989). Amount quantification, referentiality, and long wh-movement. Ms., University of Pennsylvania.

Landman, F. (2004). Indefiniteness and the Type of Sets. Oxford: Blackwell.

Landman, M. (2006). Variables in Natural Language. Ph. D. thesis, University of Massachusetts, Amherst, MA.

Matushansky, O. (2008). On the linguistic complexity of proper names. Linguistics and Philosophy 31(5), 573-627.

McNally, L. (1992). An Interpretation for the English Existential Construction. Ph. D. thesis, University of California, Santa Cruz, CA.

McNally, L. (1997). A Semantics for the English Existential Construction. New York: Garland.

Partee, B. H. (1986). Noun phrase interpretation and type-shifting principles. In J. Groenendijk, D. de Jong, and M. Stokhof (Eds.), Studies in Discourse Representation Theory and the Theory of Generalized Quantifiers, pp. 115-143. Dordrecht: Foris.

Patel-Grosz, P. and P. Grosz (2017). Revisiting pronominal typology. Linguistic Inquiry 48(2), 259-297.

Poole, E. (2017a). Movement and the Semantic Type of Traces. Ph. D. thesis, University of Massachusetts, Amherst, MA.

Poole, E. (2017b). There are no property traces. In W. G. Bennett, L. Hracs, and D. R. Storoshenko (Eds.), Proceedings of the 35th West Coast Conference on Formal Linguistics (WCCFL 35), pp. 321-330. Somerville, MA: Cascadilla Proceedings Project.

Postal, P. (1994). Contrasting extraction types. Journal of Linguistics 30, 159-186.

Romero, M. (1998). Focus and Reconstruction Effects in Wh-Phrases. Ph. D. thesis, University of Massachusetts, Amherst, MA.

Rullmann, H. (1995). Maximality in the Semantics of Wh-constructions. Ph. D. thesis, University of Massachusetts, Amherst, MA.

Sauerland, U. (1998). The Meaning of Chains. Ph. D. thesis, MIT, Cambridge, MA.

Sauerland, U. (2004). The interpretation of traces. Natural Language Semantics 12, 63-127.

Schwarz, F. (2009). Two Types of Definites in Natural Language. Ph. D. thesis, University of Massachusetts, Amherst, MA.

Williams, E. (1983). Semantic vs. syntactic categories. Linguistics and Philosophy 6, 423-446.

Williams, E. (1984). There-insertion. Linguistic Inquiry 15, 131-153.

Zamparelli, R. (2000). Layers in the Determiner Phrase. New York: Garland. 